REVISTA CIENCIAS BIOMÉ DICAS

GUÍA DE MANEJO Y PROTOCOLOS

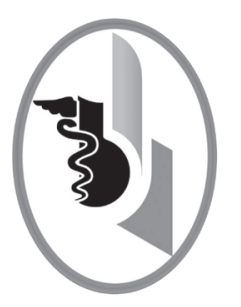

\title{
MANEJO DE VÁRICES ESOFÁGICAS EN PACIENTES CON CIRROSIS: TAMIZACIÓN, PROFILAXIS Y TRATAMIENTO DEL SANGRADO AGUDO
}

\author{
ESOPHAGEAL AND GASTRIC VARICES MANAGMENT IN \\ PATIENTS WITH CIRRHOSIS: SCREENING, PROPHYLAXIS AND \\ ACUTE BLEEDING TREATMENT
}

Yepes-Barreto |smael ${ }^{1}$

Marín-Zuluaga Juan Ignacio ${ }^{2}$

Correspondencia: ismayep@yahoo.com

Recibido para evaluación: marzo - 15 - 2015. Aceptado para publicación: octubre - 15 - 2015

\section{RESUMEN}

INTRODUCCIÓN: normalmente existen colaterales entre el sistema venoso portal y la circulación sistémica. En circunstancias normales el flujo se dirige desde las colaterales hasta la porta; sin embargo, ante la presencia de hipertensión portal este se invierte dando lugar a la aparición de várices esofagogástricas. El gold estándar para el diagnóstico es la endoscópica digestiva y todo paciente con cirrosis debe realizarse el procedimiento dentro de los 12 meses siguientes al diagnóstico de cirrosis y en un período no superior a 3 meses, si presenta una cirrosis descompensada. Las várices se clasifican como pequeñas $(<5 \mathrm{~mm})$ o grandes $(>5 \mathrm{~mm})$ en función del tamaño, debe reportarse la presencia de puntos rojos en su pared, puesto que esta información, además del cálculo del Child Pugh Turcotte, es indispensable para la estratificación del riesgo y la definición de la necesidad en el uso de profilaxis frente a las hemorragias varicosas.

Los betabloqueantes no selectivos, y la ligadura endoscópica con bandas son los dos tratamientos actualmente aceptados para la prevención de la hemorragia varicosa. La elección de cada método dependerá de las características del paciente y de los recursos disponibles. Durante la hemorragia aguda el tratamiento está dirigido a la restitución de la volemia, la prevención de las complicaciones y el uso de vasoconstrictores asplácnicos que disminuyan el flujo de las colaterales portosistémicas. En los casos de hemorragia refractaria la derivación portosistémica intrahepática (DPPI) y los shunt portosistémicos quirúrgicos pueden ser de utilidad, pero su uso es limitado dado que estos tratamientos solo se encuentran disponibles en centros hospitalarios de alta complejidad. Rev.cienc. biomed. 2015;6(2):381-389

\section{PALABRAS CLAVE}

Várices esofágicas; Cirrosis hepática; Hemorragia digestiva; Tamizaje.

\footnotetext{
1 Médico. Gastroenterólogo y Hepatólogo. Universidad de Cartagena. Pharos Centro de Investigaciones Biomédicas de la Costa. Cartagena. Colombia.

2 Médico. Internista y Hepatólogo. Hospital Pablo Tobón Uribe. Universidad Pontificia Bolivariana. Medellín. Colombia.
} 


\title{
SUMMARY
}

\begin{abstract}
Usually there are collaterals between the portal venous system and the systemic circulation. In normal circumstances the blood circulation is directed from collaterals to the portal vein; however, in the presence of portal hypertension, this is reversed resulting in the appearance of esophageal and gastric varices. The standard diagnostic is practiced with digestive system endoscopy and every patient with cirrhosis should be checked with this procedure within the twelve months following the diagnosis in a period not exceeding three months if presents a decompensated cirrhosis. The varices can be classified like small $(<5 \mathrm{~mm})$ or big $(<5 \mathrm{~mm})$ based on their size, likewise must report the presence of red spots in the wall because this information, also the Child Pugh Turcotte test, are essential for risk stratification and defining the need for the use of prophylaxis in the variceal bleeding.

The nonselective beta blockers and endoscopic elastic band ligation are the two treatments currently accepted to prevent the variceal hemorrhage. The choice of method depends on each patient characteristics and the available resources. During the acute hemorrhage the treatment has as a goal to restore the volemia, prevent the complications and the use of splanchnic vasoconstrictors to diminish the portosystemic collaterals circulation. In case of refractory bleeding, the intrahepatic portosystemic derivation and surgical portosystemic shunt can be used, but they use is limited because these treatments are only available in advanced hospitals. Rev.cienc. biomed. 2015;6(2):381-389.
\end{abstract}

\section{KEYWORDS}

Esophageal varices; Liver cirrhosis; Bleeding; Screening.

\section{INTRODUCCIÓN}

La cirrosis hepática es la principal causa de hipertensión portal en el mundo occidental. La hipertensión portal se define como el incremento del gradiente de presión venosa hepática por encima de $5 \mathrm{mmHg}$, es el punto de partida para que aparezcan las complicaciones de la cirrosis hepática $(1,2)$. Inicialmente, la presión se incrementa por una resistencia mecánica al flujo ocasionada por la distorsión de la histoarquitectura hepática; adicionalmente, existe un componente funcional ocasionado por la disminución de la producción de óxido nítrico intrahepático y la contracción de las células estrelladas que condicionan un incremento de aproximadamente el $20-30 \%$ de la resistencia hepática total (3).

Por otra parte, en el lecho vascular esplácnico existe un aumento de la producción de óxido nítrico que genera vasodilatación y aumento del flujo en este territorio vascular, ocasionando un estado de circulación hiperdinámica que, finalmente, incrementa la presión portal.

Normalmente existen colaterales entre el sistema venoso portal y la circulación sistémica, en circunstancias normales el flujo se dirige desde las colaterales hasta la porta; no obstante, ante la presencia de hipertensión portal este se invierte dando lugar a la aparición de várices en el esofagogástricas.

En pacientes con cirrosis el riesgo de desarrollar várices esofágicas de novo es de aproximadamente $7-8 \%$ al año $(4,5)$ y el riesgo de sangrado es de un $15 \%$ anual en pacientes sin historia previa de sangrado (6). En algunos pacientes este se incrementa hasta en un $60 \%$ después del primer episodio de hemorragia por hipertensión portal (7) (ver Figura No 1).

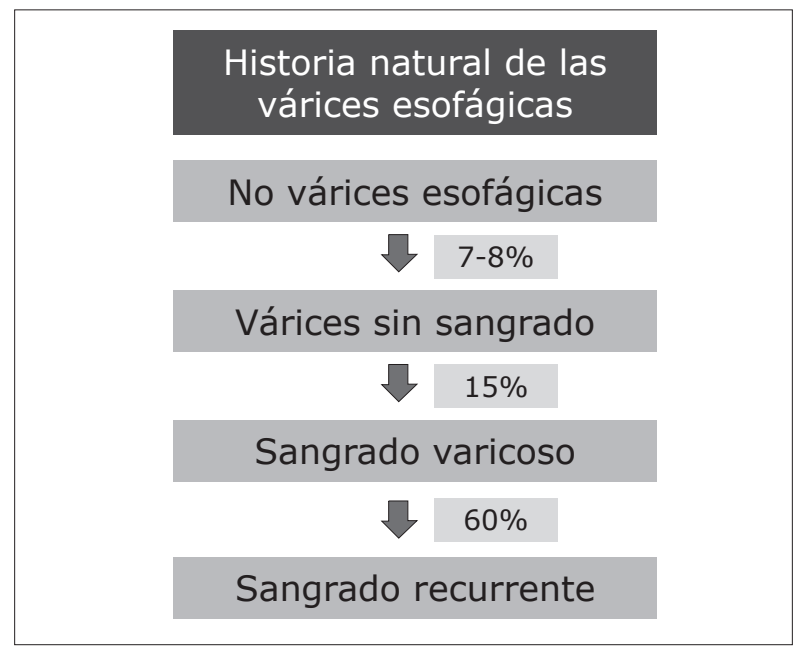

Figura No. 1. Historia natural de las várices esofágicas. 
El adecuado manejo de las várices esófago gástricas depende del grado de hipertensión portal y comprende la tamización en pacientes que aún no las hayan desarrollado, la profilaxis primaria, el tratamiento del evento hemorrágico agudo y la prevención del resangrado o profilaxis secundaria (8).

\section{TAMIZACIÓN DE VÁRICES ESOFÁGICAS}

Las várices esofágicas pueden encontrarse en aproximadamente el $50 \%$ de los pacientes con cirrosis, su aparición depende en gran parte del estadio de la enfermedad y su prevalencia puede variar desde un $40 \%$ en pacientes Child $A$, hasta un $85 \%$ en pacientes Child C (9).

El cribado sistemático se recomienda para evitar el sangrado variceal, esta es una de las complicaciones más temidas de las cirrosis por su elevada mortalidad. Aproximadamente un $20 \%$ de los pacientes fallecen dentro de las seis semanas posteriores al episodio agudo (10).

El gold estándar para el diagnóstico es la endoscópica digestiva (8) y todo paciente con cirrosis debe realizarse el procedimiento dentro de los 12 meses siguientes al diagnóstico de cirrosis y en un período no superior a tres meses si presenta una cirrosis descompensada (11). Las várices se clasifican como pequeñas $(<5 \mathrm{~mm})$ o grandes $(>5 \mathrm{~mm})$ en función del tamaño, de igual manera debe reportarse la presencia de puntos rojos en la pared esofágica o gástrica, dado que esta información es indispensable para la estratificación del riesgo y la definición del uso de la profilaxis frente a la hemorragia varicosa (8).

Las recomendaciones actuales estratifican a los pacientes en función del riesgo hemorrágico, teniendo en cuenta el estadio de la enfermedad y el control del agente etiológico para establecer los intervalos de tiempo idóneos para la búsqueda sistemática de várices en pacientes que no las hayan desarrollado a lo largo del seguimiento (12) (ver Figura $\mathrm{N}^{0} 2$ ).

De esta manera, en pacientes en los que no se haya objetivado várices esofágicas en la endoscopia inicial y presenten una enfermedad descompensada se debe realizar una

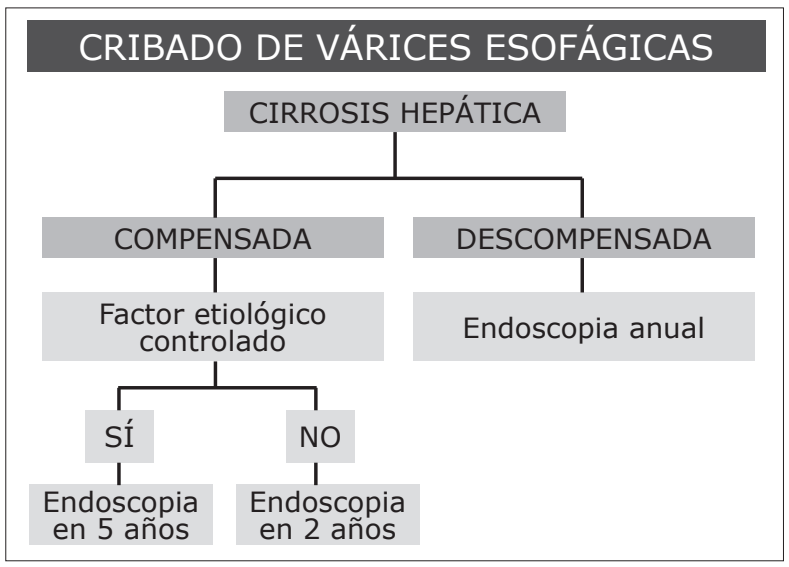

Figura No. 2. Intervalos de cribado de várices en función del estadio de la enfermedad.

endoscopia anual. El intervalo puede incrementarse a 24 meses en sujetos con enfermedad compensada en los cuales no se haya controlado el agente etiológico causal (consumo de alcohol, virus hepatotropos, síndrome metabólico, etc.) y hasta 36 meses cuando la causa de la enfermedad hepática crónica haya sido eliminada (12).

En pacientes Child $A$ con várices pequeñas y con ausencia de puntos rojos, el riesgo de sangrado es bajo, en estos casos puede optarse por continuar realizando endoscopias de cribado para vigilar el crecimiento de las várices. En estos casos, si el paciente se encuentra compensado y el agente etiológico no, se recomienda la realización de una endoscopia anual y cada dos años la aplicación de un tratamiento etiológico efectivo $(8,12)$ (ver Figura No 3).

\section{CRIBADO DE VÁRICES ESOFÁGICAS}

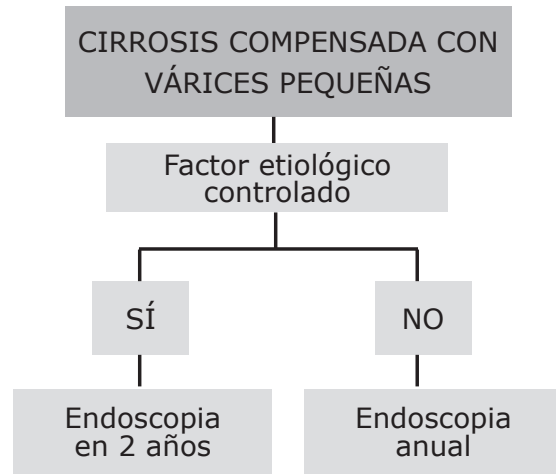

Figura No. 3. Intervalos de vigilancia endoscópica para el crecimiento de las várices en pacientes con enfermedad compensada y várices pequeñas. 
Por otra parte, el consenso de Baveno ha identificado a un grupo de pacientes en los cuales puede evitarse de forma segura la endoscopia digestiva por medio de la elastografía transitoria y el recuento plaquetario, sugiriendo que en aquellos sujetos con una elastografía transitoria $<20 \mathrm{Kpa}$ y un recuento plaquetario $>150.000$ puede evitarse la realización de la endoscopia por la baja probabilidad de presentar várices que requieran tratamiento. Estos pacientes deben seguirse anualmente y si la elastografía se incrementa (> $20 \mathrm{Kpa}$ ) o el recuento plaquetario disminuye debe realizarse el procedimiento sin dilaciones (12).

\section{PROFILAXIS PREPRIMARIA}

La profilaxis pre-primaria se practica con el fin de evitar el desarrollo de várices en el paciente con cirrosis y en aquellos que aún no la presentan.

El tratamiento con betabloqueadores no selectivos ha fallado en mostrar beneficio en estos pacientes $(5,8,12)$. Un estudio publicado en 2005 evaluó el efecto del uso de timolol en pacientes con cirrosis e hipertensión portal sobre el desarrollo de várices esofágicas de novo (5). Doscientos trece pacientes fueron aleatorizados para recibir timolol o placebo durante un tiempo mediano de 55 meses. El 39\% de los pacientes que recibieron timolol desarrollaron várices esofágicas frente a un $40 \%$ de los pacientes que recibieron placebo $(p=n s)$, aunque se observó un incremento significativo en los efectos secundarios en los pacientes asignados al grupo de tratamiento. Este estudio aporta información sólida sobre el papel actual de los betabloqueantes en la profilaxis pre-primaria y su base racional más importante, por la cual no se recomienda su uso en este grupo de pacientes.

\section{PROFILAXIS DE SANGRADO VARICEAL}

El riesgo de sangrado por várices esofagogástricas varía ampliamente en un rango que va desde el 6\% hasta el $76 \%$ anual (6). La heterogeneidad del riesgo de sangrado en estos pacientes ha motivado la búsqueda de características que nos permitan identificar a aquellos que se beneficien del inicio de la profilaxis frente a la hemorragia varicosa. Existen tres características que permiten clasificar a los pacientes en función del riesgo de sangrado: tamaño de várices, clasificación del Child Pugh Turcotte y la presencia de puntos rojos en la pared de la variz, donde el tamaño es la característica más determinante de sangrado $(1,2)$. De este modo un paciente con várices grandes, Child $\mathrm{C}$ y punto rojo tendrá un riesgo anual de sangrado del $76 \%$, contra un 6 $\%$ de sangrado anual para un paciente con várices pequeñas, Child $\mathrm{A}$ y sin puntos rojos.

\section{PROFILAXIS PRIMARIA}

Existen dos tipos de terapia para la profilaxis primaria de sangrado variceal: terapia farmacológica con betabloqueadores no selectivos o ligadura endoscópica de las várices. Las dos terapias son igualmente efectivas en la prevención del primer sangrado. Algunos estudios sugieren que los pacientes manejados con ligadura tienen menor riesgo de sangrado que los pacientes manejados con betabloqueadores, pero esto no se traduce en beneficio de mortalidad $(13,14)$. Igualmente los betabloqueadores disminuyen el riesgo de desarrollar ascitis por reducción de la hipertensión portal y también el riesgo de peritonitis bacteriana espontánea por disminuir la translocación bacteriana $(15,16)$.

El consenso de Baveno (12) clasifica los pacientes de acuerdo al riesgo de sangrado en los siguientes grupos (Tabla $\mathrm{N}^{0} 1$ ):

\begin{tabular}{|c|c|c|}
\hline \multicolumn{3}{|c|}{$\begin{array}{c}\text { TABLA No } 1 . \\
\text { PROFILAXIS DEL PRIMER SANGRADO } \\
\text { VARICEAL }\end{array}$} \\
\hline $\begin{array}{c}\text { Situación } \\
\text { clínica }\end{array}$ & Manejo & \begin{tabular}{|c} 
Seguimiento con \\
EDS
\end{tabular} \\
\hline No várices & Ninguno & Cada tres años \\
\hline $\begin{array}{l}\text { Várices } \\
\text { pequeñas } \\
\text { aisladas }\end{array}$ & Ninguno & $\begin{array}{l}\text { Cada dos } \\
\text { años si hay } \\
\text { descompensación } \\
\text { de la cirrosis. }\end{array}$ \\
\hline $\begin{array}{l}\text { Várices } \\
\text { pequeñas, } \\
\text { con punto } \\
\text { rojo y/o } \\
\text { Child B - C }\end{array}$ & Betabloqueadores & $\begin{array}{l}\text { No requiere } \\
\text { seguimiento }\end{array}$ \\
\hline $\begin{array}{l}\text { Várices } \\
\text { grandes }\end{array}$ & $\begin{array}{c}\text { Ligadura } \\
\text { endoscópica } \\
\text { con bandas o } \\
\text { betabloqueadores }\end{array}$ & $\begin{array}{l}\text { Cada seis meses } \\
\text { No requiere } \\
\text { seguimiento }\end{array}$ \\
\hline
\end{tabular}


Várices pequeñas en paciente Child A y sin punto rojo: estos pacientes tienen el menor riesgo de sangrado y podría solo hacerse seguimiento endoscópico para evaluar la aparición de puntos rojos o el crecimiento de las várices.

Várices pequeñas en pacientes Child B/C y/o con punto rojo: estos pacientes deben recibir profilaxis de sangrado con betabloqueadores no selectivos (propranolol o carvedilol). Si el paciente no tolera los betabloqueadores se debe hacer profilaxis con ligadura endoscópica.

Várices medianas y grandes: los pacientes tienen el mayor riesgo de sangrado. Deben recibir profilaxis con betabloqueadores no selectivos o ligadura endoscópica. Algunos reportes sugieren que la ligadura es más efectiva que los betabloqueadores en profilaxis, por lo que algunos la recomiendan como primera opción para estos pacientes (17).

¿Cómo utilizar los betabloqueadores en el tratamiento de la cirrosis? La meta del tratamiento farmacológico es disminuir en flujo venoso portal.

[A] Propranololes: es un betabloqueador no selectivo. La dosis de este se titula de acuerdo a la frecuencia cardiaca del paciente. Se inicia con bajas dosis (20 mg cada 12 horas) y se aumenta con la meta de una frecuencia cardiaca entre 55 y 60 latidos por minuto.

[B] Carvedilol: es un potente betabloqueador que también tiene leve bloqueo de la actividad alfa adrenérgica. Es una alternativa a los betabloqueadores no selectivos para la profilaxis de sangrado. Sin embargo, puede generar caídas más pronunciadas de la presión arterial que pueden ser mal toleradas en pacientes con cirrosis. Estos efectos son más intensos en pacientes Child B y C por la disminución del metabolismo hepático del medicamento. Por lo que la dosis recomendada en $12.5 \mathrm{mg}$ cada 12 horas para pacientes Child A y $6.25 \mathrm{mg}$ cada 12 horas para pacientes Child B y Child C

\section{MANEJO DEL SANGRADO VARICEAL AGUDO}

El sangrado por várices esofágicas representa el $85 \%$ de las causas de sangrado digestivo en los pacientes con cirrosis, y ocasiona el $30 \%$ de las muertes relacionadas con cirrosis hepática. El resultado depende del adecuado manejo y de la prevención de las complicaciones asociadas con el sangrado. Las primeras 48 a 72 horas son las de mayor riesgo y el 50 \% de todos los episodios de resangrado precoz ocurren dentro de los primeros 10 días. Después de seis semanas del episodio, la mortalidad de estos pacientes es equivalente a la de pacientes con igual Child y que nunca han sangrado (18).

Los factores asociados al resangrado temprano son: edad mayor de 60 años, etiología alcohólica de la cirrosis, sangrado inicial severo ( hemoglobina menor de $8 \mathrm{gr} / \mathrm{dL}$ ), sangrado activo al momento de la endoscopia, várices grandes y con puntos rojos, gradiente de presión de venosa hepática mayor de $20 \mathrm{mmHg}$, trombosis de la vena porta, hepatocarcinoma, trombocitopenia, encefalopatía, ascitis $(19,20)$.

Existen tres objetivos principales en el manejo: reanimación hemodinámica, tratamiento de la hemorragia y manejo de las complicaciones (Tabla N²).

\section{- Reanimación hemodinámica}

Restauración del volumen intravascular por vía intravenosa periférica de gran calibre o por línea central. La meta es lograr una PAM mayor de $70 \mathrm{mmHg}$ para garantizar la perfusión renal y de otros órganos. Se debe evitar la sobrecarga de volumen, ya que esto puede aumentar la hipertensión portal y el riesgo de resangrado (20). La meta de la hemoglobina es entre 7 y $8 \mathrm{gr} / \mathrm{dL}$ con el fin de evitar el incremento de la presión portal.

Un ensayo clínico controlado publicado en 2013 con 921 pacientes y que incluyó 277 cirróticos demostró que una estrategia restrictiva (transfundir al paciente cuando alcanza una hemoglobina menor de $7 \mathrm{gr} / \mathrm{dL}$ ) mejora significativamente los resultados en 
TABLA NO 2.

OBJETIVOS A CUMPLIR EN EL MANEJO DE SANGRADO VARICEAL

MANEJO DE SANGRADO VARICEAL

Reanimación:

PAM: $70 \mathrm{mmHg}$. Hemoglobina: $7-8 \mathrm{gr} /$

$\mathrm{dL}$

Endoscopia digestiva con ligadura de várices:

En las primeras 12 horas

Vasoconstrictor: El que esté disponible.

Somatostiantina: $250 \mathrm{mcg}$ I.V en bolo.

Seguir $250 \mathrm{mcg} /$ hora por 5 días

Octreotide: 50 mcg I.V en bolo. Seguir 25

a $50 \mathrm{mcg} /$ hora por 5 días

Terlipresina: 2 mg I.v en bolo. Seguir 1

mg I.V cada 6 horas por 5 días

Profliaxis de PBE e infección:

Si tolera v.oral: Norfloxacina $400 \mathrm{mg}$ oral cada 12 horas por 7 días

Si no tolera v.oral: Cefalosporina de 3ra generación o cubrimiento de bacilos gramnegativos entéricos dependiendo de

la microbiología local

Sangrado refractario a las medidas anteriores:

Considerar remisión a centro de mayor nivel para colocación de TIPS $(*)$

Balón de Sengstaken-Blakemore

Cirugía de salvamento, desvascularización gástrica

(*) TIPS: Shunt portosistémico transyugular

pacientes con sangrado digestivo superior incluyendo sobrevida y tasa de transfusiones (21).

La corrección de coagulopatía con transfusión de plaquetas y plasma fresco congelado solo está indicada para los procedimientos invasivos. La ligadura endoscópica en el contexto del sangrado recurrente, a pesar de un tratamiento adecuado, presenta un recuento plaquetario menor de 50.000 o un INR mayor de 1.5 .

El factor VIIa recombinante se considera en los casos que no se logra corregir la coagulopatía, a pesar de plasma fresco y plaquetas. Sin embargo, al menos dos ensayos clínicos controlados y un metaanálisis no han demostrado un claro beneficio del factor VIIa recombinante sobre la supervivencia en la hemorragia aguda, por lo que su uso en la actualidad no se recomienda (22-24).

\section{- Tratamiento de la hemorragia}

El uso de vasoconstrictores y la ligadura endoscópica de las várices son la piedra angular del control del sangrado.

\section{Vasoconstrictores}

Somatostatina, octreotide y terlipresina son los vasoconstrictores utilizados para el control del sangrado. Estos tres medicamentos fueron comparados con las dosis descritas en la Tabla 2; en un ensayo clínico controlado que incluyó 780 pacientes todos los pacientes fueron llevados a endoscopia con ligadura de várices (25). No se encontró ninguna diferencia en el éxito de hemostasia, tasa de resangrado o mortalidad. Por lo que la elección del vasoconstrictor depende de la disponibilidad y familiaridad con cualquiera de los tres medicamentos.

\section{Ligadura endoscópica de várices con bandas}

Es el procedimiento de elección para el control de várices sangrantes. Es superior a la escleroterapia en la tasa de hemostasia y se debe realizar en las primeras doce horas después del ingreso del paciente.

\section{- Prevención y tratamiento de las complicaciones}

Las complicaciones relacionadas con el sangrado y el tratamiento para la hemorragia contribuyen sustancialmente a la mortalidad por hemorragia activa. Mientras se logra la hemostasia muchos pacientes sucumben a las complicaciones que se desarrollan tras la admisión. Por lo tanto, la prevención, la vigilancia y el tratamiento son de vital importancia. Las principales complicaciones que causan la muerte son la neumonía por aspiración, sepsis, encefalopatía hepática, insuficiencia renal y el desarrollo de una falla hepática aguda sobrecrónica.

Las infecciones bacterianas se presentan hasta en el $\mathbf{2 0} \%$ de los pacientes con cirrosis que son hospitalizados con hemorragia gastrointestinal y estos pacientes tienen aumento de la mortalidad. Los sitios más comunes son las infecciones del tracto urinario, peritonitis bacteriana espontánea, las infecciones respiratorias y la bacteriemia espon- 
tánea. Múltiples estudios avalan la utilización de antibióticos profilácticos en estos pacientes, con reducción significativa de la mortalidad y del resangrado cuando se compara con placebo (Tabla 2) (26).

\section{PROFILAXIS SECUNDARIA}

El riesgo de resangrado durante los dos primeros años después de un episodio hemorrágico agudo es aproximadamente del $60 \%$ en individuos no tratados, con una mortalidad cercana al 33\% (7). Por ende, la profilaxis secundaria debe iniciarse de forma precoz y previa al alta hospitalaria.

La combinación de betabloqueadores no selectivos y la ligadura endoscópica con bandas es actualmente la estrategia más aceptada en este escenario clínico (1, 8, 12). Dos ensayos clínicos controlados y un metaanálisis han demostrado una reducción del riesgo absoluto de resangrado de aproximadamente un $25 \%$ al comparar el tratamiento combinado (nadolol + LEB) frente al tratamiento endoscópico aislado (27-29). Por ello no se recomienda la realización de LEB separado del uso de betabloqueantes en la profilaxis secundaria, salvo en el caso que existan contraindicaciones o intolerancia para la utilización de estos fármacos.

La dosis de betabloqueantes debe ser ajustada a la máxima tolerada, las sesiones de ligadura deben repetirse cada 4-6 semanas hasta la obliteración de las várices, una endoscopia de control entre 1 y 3 meses después de su desaparición y posteriormente, cada año para vigilar la reaparición de las mismas (8).

El sangrado recurrente a pesar del tratamiento de primera línea indicaría la colocación de una derivación portosistémica percutánea intrahepática (DPPI - TIPS por sus siglas en inglés) en pacientes seleccionados con buena función hepática (Child A -B) (8). La DPPI consiste en la colocación de una prótesis por vía transyugular que conecta la vena porta con una vena suprahepática reduciendo la presión en el territorio esplácnico de forma rápida y a su vez, disminuyendo el riesgo de resangrado.
Un metaanálisis que comparó la colocación de una DPPI con la LEB como terapias de primera línea frente al resangrado varicoso (30) observó una disminución del sangrado en el primer grupo a expensas de un mayor riesgo de presentar encefalopatía hepática y sin beneficios, sobre la supervivencia de los pacientes. En la actualidad la colocación de la DPPI se recomienda únicamente en la profilaxis secundaria cuando falla el tratamiento combinado de betabloqueantes y LEB.

Los shunts portosistémicos quirúrgicos son también muy efectivos en la prevención del resangrado, con un perfil de eficacia y seguridad muy similar al ser comparados con la DPPI después del fallo de la terapia estándar. La elección del tipo de tratamiento derivativo dependerá de la experiencia y de los recursos locales disponibles (31).

\section{CASO CLÍNICO}

Varón de 58 años con obesidad mórbida y diabetes mal controlada, diagnosticado con hígado graso desde hace aproximadamente 10 años consultó por dolor abdominal y tinte ictérico de mucosas. Bajo la sospecha de colecistitis se realizó una ecografía abdominal que mostró hígado de bordes lobulados y superficie micronodular. Se descartaron las etiologías virales, autoinmunes y enfermedades de depósito por enfermedad hepática crónica, y se realizó un diagnóstico de cirrosis por esteatohepatitis no alcohólica en estadio Child Pugh A. Se realizó una endoscopia inicial que no mostró presencia de várices, luego se programaron revisiones ecográficas y analíticas cada seis meses.

Durante el seguimiento posterior el paciente presentó deterioro de la función hepática (Child Pugh B) y desarrolló várices grandes sin puntos rojos, por lo que se inició tratamiento profiláctico. Tres años después de la aparición de las várices el paciente presentó su primer episodio hemorrágico que se trató con ligadura y octeotride con lo que se obtuvo buena evolución.

CONFLICTOS DE INTERESES: ninguno que declarar.

FINANCIACIÓN: recursos propios de los autores. 


\section{REFERENCIAS BIBLIOGRÁFICAS}

1. de Franchis R. Evolving consensus in portal hypertension. Report of the Baveno IV consensus workshop on methodology of diagnosis and therapy in portal hypertension. J Hepatol 2005;43:167-176.

2. Ripoll C, Groszmann R, Garcia-Tsao G, Grace N, Burroughs A, Planas R, Escorsell A, et al. Hepatic venous pressure gradient predicts clinical decompensation in patients with compensated cirrhosis. Gastroenterology 2007;133:481-488.

3. Bhathal PS, Grossman HJ. Reduction of the increased portal vascular resistance of the isolated perfused cirrhotic rat liver by vasodilators. J Hepatol 1985;1:325-337.

4. Merli M, Nicolini G, Angeloni S, Rinaldi V, De Santis A, Merkel C, Attili AF, et al. Incidence and natural history of small esophageal varices in cirrhotic patients. J Hepatol 2003;38:266-272.

5. Groszmann RJ, Garcia-Tsao G, Bosch J, Grace ND, Burroughs AK, Planas R, Escorsell A, et al. Beta-blockers to prevent gastroesophageal varices in patients with cirrhosis. N Engl J Med 2005;353:2254-2261.

6. Prediction of the first variceal hemorrhage in patients with cirrhosis of the liver and esophageal varices. A prospective multicenter study. N Engl J Med 1988;319:983-989.

7. D'Amico G, Pagliaro L, Bosch J. Pharmacological treatment of portal hypertension: an evidence-based approach. Semin Liver Dis 1999;19:475-505.

8. Garcia-Tsao G, Sanyal AJ, Grace ND, Carey W. Prevention and management of gastroesophageal varices and variceal hemorrhage in cirrhosis. Hepatology 2007;46:922-938.

9. Navasa M, Pares A, Bruguera M, Caballeria J, Bosch J, Rodes J. Portal hypertension in primary biliary cirrhosis. Relationship with histological features. J Hepatol 1987;5:292298.

10. El-Serag HB, Everhart JE. Improved survival after variceal hemorrhage over an 11-year period in the Department of Veterans Affairs. Am J Gastroenterol 2000;95:3566-3573.

11. Kanwal F, Kramer J, Asch SM, El-Serag H, Spiegel BM, Edmundowicz S, Sanyal AJ, et al. An explicit quality indicator set for measurement of quality of care in patients with cirrhosis. Clin Gastroenterol Hepatol 2010;8:709-717.

12. de Franchis R. Expanding consensus in portal hypertension: Report of the Baveno VI Consensus Workshop: Stratifying risk and individualizing care for portal hypertension. J Hepatol 2015;63:743-752.

13. Li L, Yu C, Li Y. Endoscopic band ligation versus pharmacological therapy for variceal bleeding in cirrhosis: a meta-analysis. Can J Gastroenterol 2011;25:147-155.

14. Triantos CK, Burroughs AK. Prevention of the development of varices and first portal hypertensive bleeding episode. Best Pract Res Clin Gastroenterol 2007;21:31-42.

15. Hernandez-Gea V, Aracil C, Colomo A, Garupera I, Poca M, Torras X, Minana J, et al. Development of ascites in compensated cirrhosis with severe portal hypertension treated with beta-blockers. Am J Gastroenterol 2012;107:418-427.

16. Senzolo M, Cholongitas E, Burra P, Leandro G, Thalheimer U, Patch D, Burroughs AK. beta-Blockers protect against spontaneous bacterial peritonitis in cirrhotic patients: a meta-analysis. Liver Int 2009;29:1189-1193.

17. Vlachogiannakos J, Goulis J, Patch D, Burroughs AK. Review article: primary prophylaxis for portal hypertensive bleeding in cirrhosis. Aliment Pharmacol Ther 2000;14:851-860.

18. Prandi D, Rueff B, Roche-Sicot J, Sicot C, Maillard JN, Benhamou JP, Fauvert R. Lifethreatening hemorrhage of the digestive tract in cirrhotic patients. An assessment of the postoperative mortality after emergency portacaval shunt. Am J Surg 1976;131:204209.

19. Cerqueira RM, Andrade L, Correia MR, Fernandes CD, Manso MC. Risk factors for inhospital mortality in cirrhotic patients with oesophageal variceal bleeding. Eur J Gastroenterol Hepatol 2012;24:551-557.

20. Krige JE, Kotze UK, Distiller G, Shaw JM, Bornman PC. Predictive factors for rebleeding and death in alcoholic cirrhotic patients with acute variceal bleeding: a multivariate analysis. World J Surg 2009;33:2127-2135.

21. Villanueva C, Colomo A, Bosch A, Concepcion M, Hernandez-Gea V, Aracil C, Graupera $\mathrm{I}$, et al. Transfusion strategies for acute upper gastrointestinal bleeding. N Engl J Med 2013;368:11-21.

22. Bosch J, Thabut D, Bendtsen F, D'Amico G, Albillos A, Gonzalez Abraldes J, Fabricius S, et al. Recombinant factor VIIa for upper gastrointestinal bleeding in patients with cirrhosis: a randomized, double-blind trial. Gastroenterology 2004;127:1123-1130.

23. Bosch J, Thabut D, Albillos A, Carbonell N, Spicak J, Massard J, D'Amico G, et al. Recombinant factor VIIa for variceal bleeding in patients with advanced cirrhosis: A randomized, controlled trial. Hepatology 2008;47:1604-1614.

24. Marti-Carvajal AJ, Karakitsiou DE, Salanti G. Human recombinant activated factor VII for upper gastrointestinal bleeding in patients with liver diseases. Cochrane Database Syst Rev 2012;3:CD004887. 
25. Seo YS, Park SY, Kim MY, Kim JH, Park JY, Yim HJ, Jang BK, et al. Lack of difference among terlipressin, somatostatin, and octreotide in the control of acute gastroesophageal variceal hemorrhage. Hepatology 2014;60:954-963.

26. Chavez-Tapia NC, Barrientos-Gutierrez T, Tellez-Avila F, Soares-Weiser K, Mendez-Sanchez N, Gluud C, Uribe M. Meta-analysis: antibiotic prophylaxis for cirrhotic patients with upper gastrointestinal bleeding - an updated Cochrane review. Aliment Pharmacol Ther 2011;34:509518.

27. de la Pena J, Brullet E, Sanchez-Hernandez E, Rivero M, Vergara M, Martin-Lorente JL, Garcia Suarez C. Variceal ligation plus nadolol compared with ligation for prophylaxis of variceal rebleeding: a multicenter trial. Hepatology 2005;41:572-578.

28. Lo GH, Lai KH, Cheng JS, Chen MH, Huang HC, Hsu PI, Lin CK. Endoscopic variceal ligation plus nadolol and sucralfate compared with ligation alone for the prevention of variceal rebleeding: a prospective, randomized trial. Hepatology 2000;32:461-465.

29. Gonzalez R, Zamora J, Gomez-Camarero J, Molinero LM, Banares R, Albillos A. Meta-analysis: Combination endoscopic and drug therapy to prevent variceal rebleeding in cirrhosis. Ann Intern Med 2008;149:109-122.

30. Papatheodoridis GV, Goulis J, Leandro G, Patch D, Burroughs AK. Transjugular intrahepatic portosystemic shunt compared with endoscopic treatment for prevention of variceal rebleeding: A meta-analysis. Hepatology 1999;30:612-622.

31. Henderson JM, Boyer TD, Kutner MH, Galloway JR, Rikkers LF, Jeffers LJ, Abu-Elmagd K, et al. Distal splenorenal shunt versus transjugular intrahepatic portal systematic shunt for variceal bleeding: a randomized trial. Gastroenterology 2006;130:1643-1651.

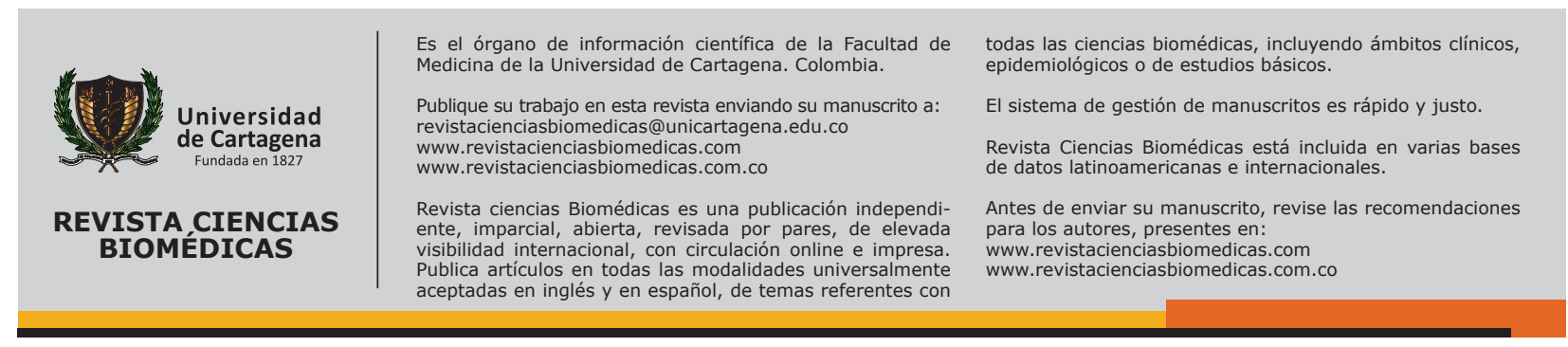

